Case Report

\title{
Congenital tuberculosis presenting with erythema nodosum: a case report
}

\author{
NS Joshi, R Sinha, A Lois, S Save \\ Sri Lankan Journal of Infectious Diseases 2022 Vol.12(1):E3 1-5 \\ DOI: http://dx.doi.org/10.4038/sljid.v12i1.8398
}

\begin{abstract}
:
Erythema nodosum is an entity with a relatively low incidence of 1 to 5 per 100,000 live births. The condition is relatively uncommon in prepubertal children and exceptionally rare before 2 years of age. In the paediatric age group, both sexes are equally affected. Although uncommon in children, it remains the most frequent form of panniculitis in paediatrics for which the diagnosis is almost always clinical. The most common cause in children is streptococcal pharyngitis. The association of erythema nodosum with tuberculosis is well known in endemic areas and must always be excluded. All children with erythema nodosum should be stratified by risk for tuberculosis exposure. Congenital tuberculosis is an unusual and severe clinical presentation of tuberculosis infection. It is rare, even where tuberculosis is endemic. Timely administration of standard antitubercular treatment results in a good outcome.

We report a two-month-old male infant who presented with fever, lethargy, dyspnea, and refusal to feed and whose mother was diagnosed as probable multidrug-resistant tuberculosis. Our patient emphasizes the importance of maternal history, typical skin lesions, and histopathological study in order to approach a definitive diagnosis and institute timely administration of antitubercular drugs.
\end{abstract}

Keywords: erythema nodosum, congenital tuberculosis, infant, significant maternal history

\section{Introduction}

Congenital tuberculosis (TB) is rare, even where tuberculosis is endemic. ${ }^{1} \mathrm{~TB}$ is relatively common in pregnant women, the prevalence of active TB in pregnant and postpartum women from high burden countries being up to 60 cases per 100000 population per year. ${ }^{2}$ In contrast, in low burden TB countries, the prevalence is lower than 20 cases per 100000 population per year. ${ }^{2}$ Till 1989 only 300 cases were reported in the scientific literature. Subsequently, 58 cases were reported

${ }^{1}$ Topiwala National Medical College, Mumbai, India

Address for correspondence: Dr. Sushma Save, Ajmera AEON, 2002, North (B) Wing, Bhakti Park, Wadala (East), Mumbai 400037, India. Telephone: +00919819096819 E-mail: sushmasave73@gmail.com

https://orcid.org/0000-0002-6498-7962

Received 16 May 2021 and revised version accepted 31 December 2021. Published 7 February 2022

(c) (†)

use, distribution, and reproduction in any medium, provided the original author and source are credited. 
in 1994, and from 2001 to December 2005, 18 more cases were mentioned. ${ }^{2}$ Erythematous and tender nodules, even in an atypical site, suggested the disease in the patient presented here. We present a 2-month-old baby who presented with erythematous and tender nodules in an atypical site and a maternal history of tuberculosis.

\section{Case report:}

A two-month-old male child, firstborn of a second-degree consanguineous marriage, presented with moderate grade fever for seven days, difficulty in breathing, refusal to feed, and lethargy for two days. During the antenatal period, his mother was diagnosed to have smear-positive pulmonary tuberculosis. Anti-tubercular therapy (ATT) with first-line drugs was initiated. Second-line drugs were started in view of no clinical response, and she was treated as a patient with multi-drugresistant tuberculosis. The mother conceived at the sixth month of treatment. Her baby was born by normal vaginal delivery at full term. Placental samples were not tested for tuberculosis. His birth weight was $2.8 \mathrm{~kg}$ and he cried immediately after birth. He was started on oral isoniazid $(10 \mathrm{mg} / \mathrm{kg})$. However, the treatment was discontinued by his parents. The infant had received birth doses of BCG, Hep B, and OPV.

On physical examination, the baby was ill-looking, febrile, and had tachycardia, tachypnoea, respiratory distress, and pallor. On respiratory system examination, bilateral crepitations were present. On abdominal examination, hepatomegaly was present. Cardiovascular and central nervous system examinations were normal. Neonatal reflexes were normal. In view of respiratory

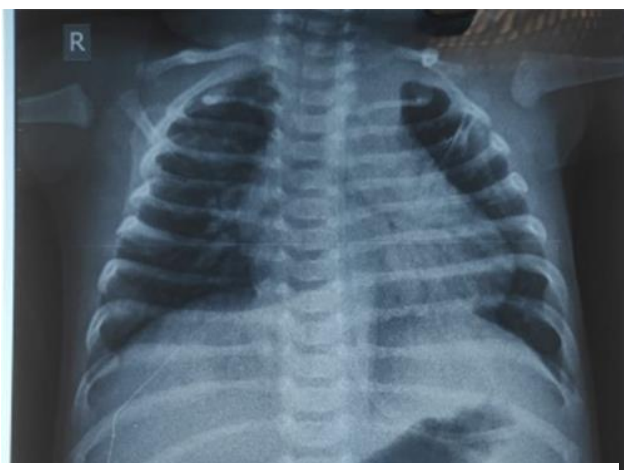

Figure 1: X-ray chest showed right sided consolidation

The patient required intensive care management and mechanical ventilation in view of impending respiratory failure. He had a stormy clinical course with respiratory failure, severe metabolic acidosis, and profound arterial hypotension unresponsive to aggressive fluid resuscitation and inotropes. HRCT Thorax (Figure 2) was suggestive of bilateral necrotizing lymph nodes in the perihilar region and superior segments of the lower lobes, and nonenhancing lesion in segment 8 of the liver.

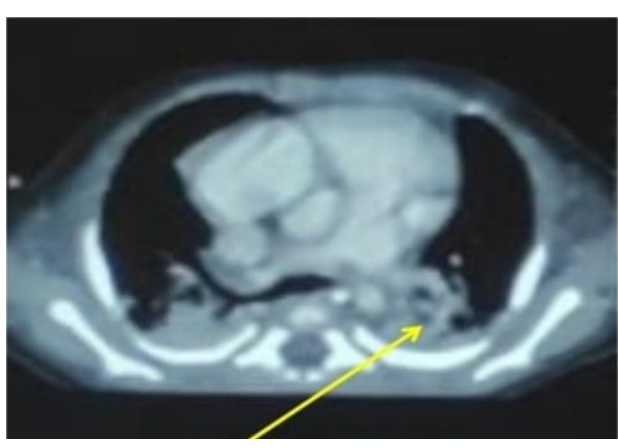

Figure 2

HRCT Thorax showing bilateral necrotising lymphadenopathy 
Ultrasonography (USG) of the abdomen revealed significant inguinal lymphadenopathy, with two left inguinal lymph nodes measured at 1.2 to $1.6 \mathrm{~cm}$.

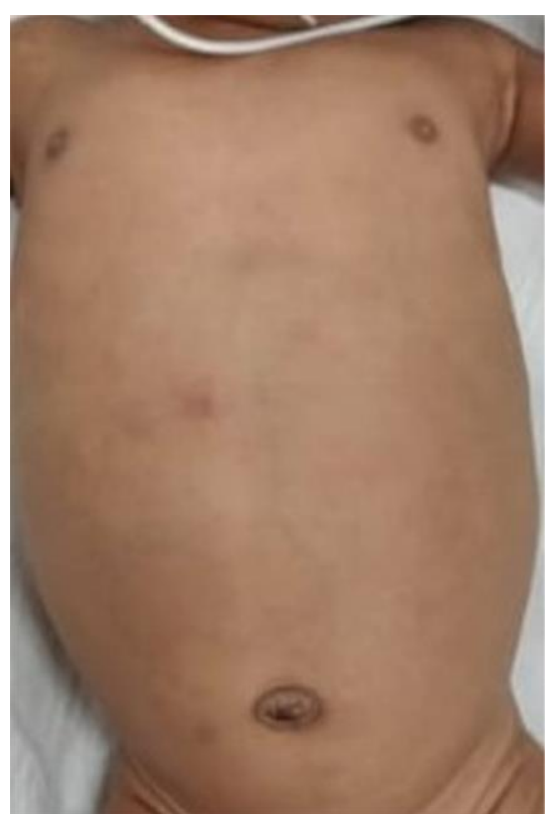

Figure 3: Clinical photograph of erythematous lesions over abdomen
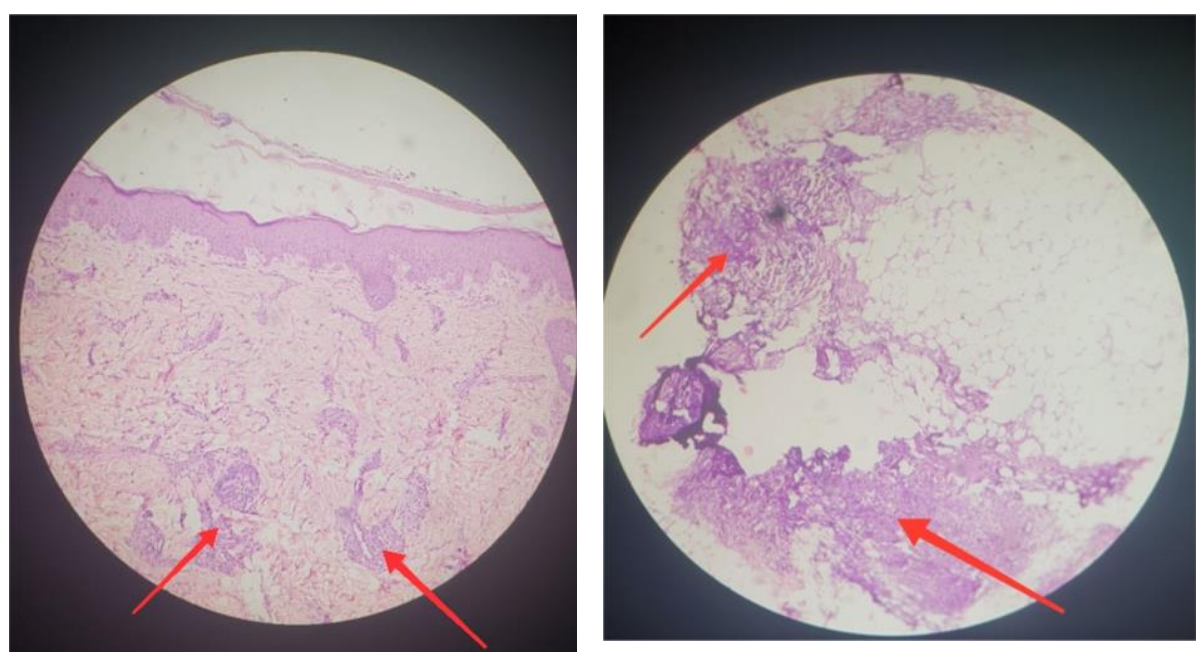

Figure 4: Histopathology image of skin showing perivascular lymphocytic infiltrate and septal panniculitis.
In view of the clinical profile, significant maternal history, inguinal lymphadenopathy, hepatomegaly with a nonenhancing lesion in the liver, and significant radiological findings, congenital tuberculosis was strongly suspected. The baby was started on a conventional regimen for MDR tuberculosis as the mother was diagnosed with MDR TB during pregnancy. The intensive phase comprised of levofloxacin (20mg/kg/day), ethionamide (20 $\mathrm{mg} / \mathrm{kg} /$ day $), \quad$-aminosalicylic acid $(150 \mathrm{mg} / \mathrm{kg} /$ day $)$, ethambutol (25 $\mathrm{mg} / \mathrm{kg} /$ day) and pyrazinamide (30 $\mathrm{mg} / \mathrm{kg} /$ day).

Blood and CSF cultures showed no growth. The patient developed three erythematous lesions on the abdominal wall and on the anterior aspect of his lower limbs (Figure $3)$. These lesions were ill-defined, warm, round, and without ulceration. The nodules were initially bright to deep red and resolved within weeks. Skin biopsy was done, and histopathology was suggestive of perivascular infiltrate and septal panniculitis suggestive of erythema nodosum (Figure 4).

The patient improved clinically and was extubated. He was discharged after 17 days of hospital stay on anti-tubercular therapy and parents were advised to continue intensive phase therapy for six months. They were also advised to attend a follow-up clinic every month during treatment and after treatment six monthly for two years.

The timeline of the case is shown in Table 1. 


\begin{tabular}{|l|l|}
\hline JUNE 19 & $\begin{array}{l}\text { Admitted to hospital with 7 days' fever and 2 days of difficulty in breathing, refusal to feed, and lethargy. The } \\
\text { patient was kept on O2 by nasal prongs @ 2 litres/min. IV Amoxiclav (100 mg/kg/day) begun. }\end{array}$ \\
\hline DAY 2 & $\begin{array}{l}\text { Required intubation mechanical ventilation. Was started on antitubercular drugs. } \\
\text { Antibiotics escalated to IV Meropenem (120mg/kg/day) and IV Vancomycin }(60 \mathrm{mg} / \mathrm{kg} / \mathrm{day}) .\end{array}$ \\
\hline DAY 3 & Required ionotropic support. \\
\hline DAY 6 & $\begin{array}{l}\text { HRCT Thorax was suggestive of bilateral necrotizing lymph nodes in the perihilar region and in superior } \\
\text { segments of lower lobes, non-enhancing lesion in segment } 8 \text { of the liver. } \\
\text { USG abdomen revealed significant inguinal lymphadenopathy, two left inguinal lymph nodes measured } 1.2 \text { to } \\
1.6 \mathrm{~cm} .\end{array}$ \\
\hline DAY 10 & The child was extubated. \\
\hline DAY 14 & The child developed three erythematous lesions on the abdomen and on the anterior aspect of lower limbs. \\
\hline DAY 15 & A skin biopsy was performed. \\
\hline DAY 17 & The patient was discharged after 17 days of hospital stay on antitubercular therapy. \\
\hline
\end{tabular}

Table 1: Timeline of the case.

\section{Discussion}

Congenital tuberculosis occurs due to exposure of an infant to Mycobacterium tuberculosis during the intrauterine period or during normal birth. Transmission of maternal TB to the fetus can be either transplacental or by aspiration of infected amniotic fluid. ${ }^{1}$ The most common clinical manifestations are hepatosplenomegaly (76\%), respiratory distress (72\%), fever (48\%), and lymphadenopathy (32\%). ${ }^{1}$ This case highlights the importance of obtaining a history of maternal $\mathrm{TB}$ and the typical radiological pattern for early diagnosis of congenital or neonatal TB which in turn facilitates prompt treatment and a good outcome.

The standardized criteria for distinguishing congenital tuberculosis from postnatally acquired tuberculosis were first established by Beitzke in 1935 and later revised by Cantwell in 1994. The infant must have proven tuberculosis lesions and at least one of the following: 1) lesions in the first week of life 2) a primary hepatic complex or caseating hepatic granulomas 3) maternal genital tract or placental tuberculosis 4) exclusion of the possibility of postnatal transmission by thorough investigation of contacts. ${ }^{1}$ The possibility of postnatal transmission was ruled out by doing a diagnostic workup for tuberculosis of the contacts which were negative.

Congenital tuberculosis should be considered in the differential diagnosis in newborns who have 1) nonresponsive or worsening pneumonia in regions with high rates of tuberculosis,

2) nonspecific symptoms but mother has a diagnosis of tuberculosis, 3) fever and hepatosplenomegaly and 4) high lymphocyte count in the cerebrospinal fluid without an identified bacterial pathogen.

The index patient fulfilled the first, second, and third criteria. ${ }^{3}$ Complications of late diagnosis of congenital tuberculosis include meningitis, miliary tuberculosis, and otitis media, resulting in seizures, deafness, and death. ${ }^{3}$

In view of significant maternal history, worsening pneumonia, poor clinical response, necrotizing lymph nodes in bilateral perihilar region and in superior segments of lower lobes, and a nonenhancing lesion in segment 8 of the liver seen in the HRCT thorax, congenital tuberculosis was strongly suspected and antitubercular drug treatment started. The infant showed a good response to the same. 
Erythema nodosum are tender, erythematous, subcutaneous nodules symmetrically located on the shins, ankle, and knees. The nodules usually measure 1 to $5 \mathrm{~cm}$ and are usually bilaterally distributed. It is due to delayed type 4 hypersensitivity reaction to mycobacterial antigens. The histopathological examination of the skin lesions of this patient showed superficial and deep perivascular lymphocytic infiltrate in the overlying dermis and septal panniculitis which was suggestive of erythema nodosum. ${ }^{4,5}$ It usually has spontaneous resolution within 3 to 6 weeks without leaving a scar or atrophy of the skin. ${ }^{6}$

\section{Conclusion}

Congenital tuberculosis is a severe infection and delayed treatment results in a poor outcome. A high degree of suspicion is required towards diagnosing tuberculosis in pregnancy. The nonspecific nature of signs and symptoms of tuberculosis in pregnancy and infancy makes early detection challenging. Newborns with pneumonia unresponsive to aggressive antibiotic therapy and usually diagnosed as sepsis should be aggressively investigated for congenital tuberculosis. Overwhelming sepsis can occur in the presence of tuberculosis. Screening for tuberculosis should be part of antenatal care at the slightest suspicion of tuberculosis. ${ }^{7}$ It is extremely important to ensure that MDR TB is appropriately diagnosed and treated in infants and children.

\section{Declarations}

Conflict of Interest - none

Acknowledgment - The authors thank Dr Ramesh Bharmal, Dean - T.N. Medical College \& BYL Nair Hospital for granting permission to publish this manuscript

Funding: None

Ethics statement - Ethical approval was not required for this manuscript.

Author contributions - Preparation of first draft: NJ, RS, AL; Collection of data: NJ, RS, SS, AL; Interpretation of data: SS, NJ, RS.; Literature Search: NJ, RS, AL; Conceptualization: SS, RS, NJ; Intellectual inputs for improvement of Manuscript: SS, RS, NJ; Approval of Final Draft: SS, RS, NJ

\section{References}

1. Chang $\mathrm{CW}, \mathrm{Wu} \mathrm{PW}$, Yeh $\mathrm{CH}$ et al. Congenital tuberculosis: case report and review of the literature. Paediatrics and International Child Health. 2018; 38(3):216-9. doi: https://doi.org/10.1080/20469047.2017.1315912

2. Saramba MI, Zhao D. A perspective of the diagnosis and management of congenital tuberculosis. Journal of Pathogens. 2016; Article Id 8623825. doi: https://doi.org/10.1155/2016/8623825

3. Peterside O, Adeyemi OO, Kunle-Olowu OE et al. Congenital tuberculosis: a case report and review of the literature. Nigerian Journal of Paediatrics 2013; 40(1):93-6. doi: 10.4314/njp.v40i1.20

4. Requena L, Requena C. Erythema nodosum. Dermatology Online Journal, 2002; 8(1). doi: https://doi.org/10.5070/D34829t6rn

5. Schwartz RA, Nervi SJ. Erythema nodosum: a sign of systemic disease. American Family Physician 2007; 75(5):695-700. PMID: 17375516 doi: 10.1007/s12519-018-0191-1

6. Bastos F, Pérez LT, Narváes CP et al. Panniculitis in the newborn: a case report. Einstein (São Paulo). 2011; 9(4):534-7. doi: 10. https://doi.org/10.1590/S1679-45082011RC2091

7. Vilarinho LC. Congenital tuberculosis: a case report. Brazilian Journal of Infectious Diseases 2006; 10(5):368-70. doi: 10.1590/s1413-86702006000500014. 